\title{
Effects of sensitive electrical stimulation based cueing in Parkinson's disease: a preliminary study
}

\author{
Benoît Sijobert (1), Christine Azevedo-Coste (1), David Andreu (1), Claudia Verna (2), \\ Christian Geny (2)
}

(1) INRIA - LIRMM Université de Montpellier, Montpellier, France; (2) CHU Montpellier, Montpellier, France

This article is distributed under the terms of the Creative Commons Attribution Noncommercial License (CC BY-NC 4.0) which permits any noncommercial use, distribution, and reproduction in any medium, provided the original author(s) and source are credited.

\begin{abstract}
This study aims to investigate the effect of a sensitive cueing on Freezing of Gait (FOG) and gait disorders in subjects suffering from Parkinson's disease (PD). 13 participants with Parkinson's disease were equipped with an electrical stimulator and a foot mounted inertial measurement unit (IMU). An IMU based algorithm triggered in real time an electrical stimulus applied on the arch of foot at heel off detection. Starting from standing, subjects were asked to walk at their preferred speed on a path comprising $5 \mathrm{~m}$ straight, u-turn and walk around tasks. Cueing globally decreased the time to achieve the different tasks in all the subjects. In "freezer" subjects, the time to complete the entire path was reduced by $19 \%$. FOG events occurrence was lowered by $12 \%$ compared to baseline before and after cueing. This preliminary work showed a positive global effect of an electrical stimulation based cueing on gait and FOG in PD.
\end{abstract}

Key Words: Freezing of gait, Parkinson's disease, electrical stimulation, cueing, gait analysis, inertial sensors

Eur J Transl Myol 2016; 26 (2): 122-128

Parkinson's disease (PD) is the second most common neurodegenerative disorder. It affects approximately ten million people worldwide, among them essentially adults over 60 years old. ${ }^{12}$ The number of persons suffering from PD has been increasing with the aging population. Various symptoms having an important impact on quality of life are related to $\mathrm{PD}$, such as tremor, bradykinesia and gait impairments leading sometimes to falls. ${ }^{3}$ Individuals with advanced Parkinson's disease can be subject to a specific paroxysmal symptom called freezing of gait (FOG) and defined by Heremans ${ }^{4}$ as "a brief, episodic absence or marked reduction of forward progression of the feet despite the intention to walk". FOG can appear in different daily life situations, such as gait initiation, turning back, standing up from a chair, changes in floor patterns or going through narrow spaces. ${ }^{5-7}$ Previous works have shown that visual or auditory stimuli can help individuals with PD to reduce the occurrence and duration of FOG events ${ }^{8-10}$ thereby improving their gait. ${ }^{11,12}$ In their meta-analysis, Spaulding et al. $^{13}$ reviewed the numerous studies on visual and auditory cueing by comparing their efficacy on gait from 25 chosen articles. Evaluating velocity, stride length and cadence, they demonstrated a positive influence on these 3 kinematic variables with auditory cueing, while visual cueing only resulted in stride length's changes.

To our knowledge, only two studies used electrical stimulation (ES) applied on individuals suffering from PD. Mann et al. ${ }^{14}$ studied the feasibility of functional electrical stimulation to assist gait in PD. During eight weeks they applied stimulation of the common peroneal nerve of the more affected side on 6 subjects. Through electrodes positioned over the head of the fibula and the motor point of the tibialis anterior muscle, the stimulation was triggered by a pressuresensitive switch in the shoe and set to gain effective dorsiflexion and eversion of the foot during walking similarly to a drop foot stimulation modality. An immediate improvement was demonstrated with FES on distance and average stride length during a 3-min walk but not on number of steps and walking speed. Fewer episodes of FOG occurred during the treatment period. In Djurić-Jovičić et al. ${ }^{15}$, a similar stimulation approach was applied on nine PD subjects. Peroneal nerve was stimulated during the swing phase on the weakest side. Results showed a decreased duration of double support phase and variability of stride duration and stride length with FES. Two subjects did not experience motor blocks in a few places along the path 


\section{Sensitive electrical stimulation based cueing in Parkinson's disease}

Eur J Transl Myol 26 (2): 122-128

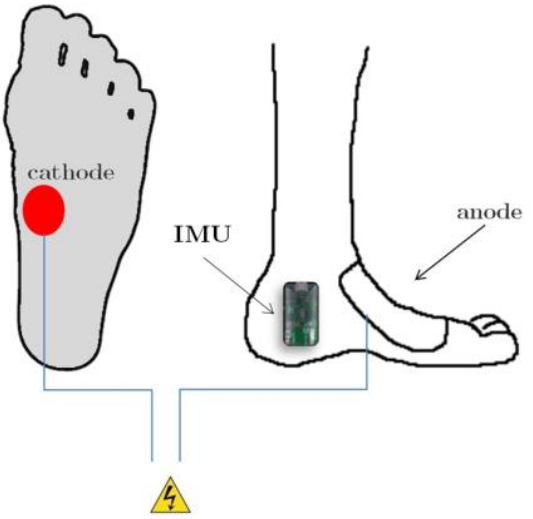

Fig 1. Electrodes location used for electrical stimulation. To deliver stimulation, a selfadhesive electrode $\left(2.6 \mathrm{~cm}^{2}\right)$ is placed as cathode on arch of the foot and a large common anode on dorsum of the foot.

where they otherwise had problems with FOG. From the previous statements, we decided to design a protocol based on electrical stimuli cueing.

In both on and off conditions (under medication or not), turning phase has been demonstrated as the most frequent trigger of freezing of gait in Parkinson's disease. ${ }^{16}$ This is also what we observed in our experiments. ${ }^{17}$ Plotnik et al. tried to explain this occurrence by the asymmetric nature of the task which would increase interlimb synchronization difficulties ${ }^{18}$ Crenna et al. showed it could possibly be related to head rotation. Patients in the early stage of the disease starting their head rotation later than controls while turning. ${ }^{19}$ In Nieuwboer et al. $^{20}$, authors chose to focus their work on different cueing modalities on turn speed only. In addition, as shown $\mathrm{in}^{21}$, evaluating freezing of gait poses difficulties as its likelihood to happen highly relies on environmental triggers, cognitive input and medication. In order to increase FOG occurrence during experimentations, we designed an experimental path including a maximum of turning phases. Numerous studies confirmed Parkinson's disease motor deficits are associated with proprioceptive impairment. In
Vaugoyeau et al. work $^{22}$, the authors subjected standing subjects to small angular sinusoidal perturbations applied to a supporting platform and asked them to maintain verticality. In the absence of visual cues, the PD subjects were clearly unable to use proprioceptive information as feedback to control their body verticality and stabilize the body segments, resulting in blocking head and shoulders. The same strategies have been observed during their gait. ${ }^{23}$ The authors concluded sensorimotor integration deficits partly account for the postural and locomotion impairments observed in PD. Using muscle vibration on the trajectories of voluntary dorsiflexion movements of the ankle joint, Khudados et al. ${ }^{24}$ showed that proprioceptive regulation of voluntary movement is disturbed in PD. El-Tamawy et al. ${ }^{25}$ used augmented proprioceptive cues during gait on thirty levodopa-dependent PD subjects. They applied vibratory stimuli to the feet plantar surfaces (below the heel and forefoot) through miniature hidden vibrating devices that sent rhythmic vibrations to the skin synchronized with the step in the push off-phase of the gait. Results demonstrated a significant improvement in gait kinematics and angular excursion of lower limb joints. Similarly, Kleiner et al. ${ }^{26}$ applied mechanical stimulation (AMPS: Automated Mechanical Peripheral Stimulation Treatment) on four specific target areas in patient's feet while the subject was laid down and reported a $15 \%$ improvement in gait velocity after treatment.

\section{Materials and Methods}

\section{Stimulation}

Inspired by Spaich work on hemiparetic gait, ${ }^{26,27}$ we stimulated the arch of the foot as shown in Fig. 1. The stimulation pattern consists in five $1 \mathrm{~ms}$-wide biphasic pulses delivered at $200 \mathrm{~Hz}$, repeated 4 times at $15 \mathrm{~Hz}$. Current amplitude was adjusted in order for the subject to feel the stimulation without any discomfort. Patients with advanced Parkinson's disease are usually subject to altered gait patterns which makes difficult to segment and to reliably detect gait events or compute

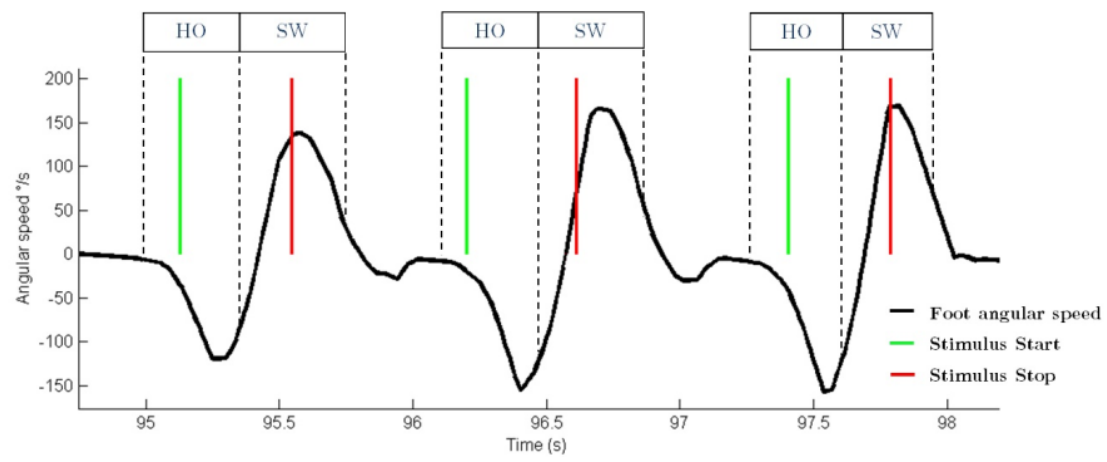

Fig 2. Example of stimulation triggering based on Equ 2 from an experimental record. In green and red are respectively the start and stop actual stimulation events triggered by the non-stationary period detection algorithm. Heel off $(\mathrm{HO})$ and swing $(\mathrm{SW})$ phases were manually added to the figure from literature for reference 


\section{Sensitive electrical stimulation based cueing in Parkinson's disease}

Eur J Transl Myol 26 (2): 122-128
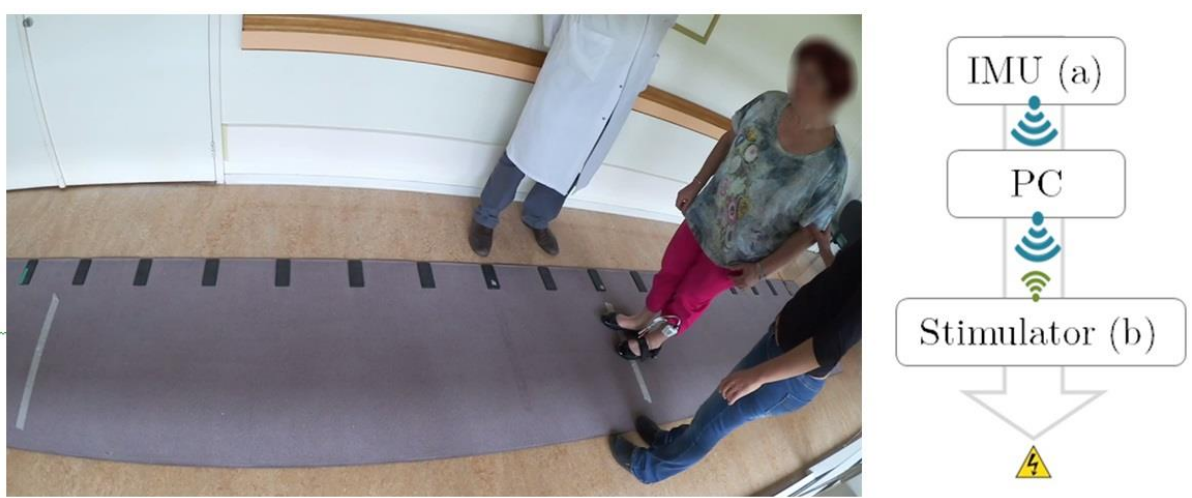

Fig 3. The subject is equipped with an inertial measurement unit (a) and a wirelessly programmable stimulator (b) connected through a PC.

gait parameters compared to healthy subjects. Different methods have been proposed based on wearable and non-wearable systems using multiple sensors, ${ }^{28}$ but our aim has been to conceive a ready-to-use patient-oriented solution, ${ }^{29}$ using a minimum amount of sensors and not requiring individual calibration or threshold parameterization for correctly triggering stimulation. In Moore et al. work, ${ }^{5}$ the investigators monitored during 75 min subjects suffering from Parkinson. For detecting locomotor activity, they defined periods where the RMS vertical acceleration was greater than $0.4 \mathrm{~m} / \mathrm{s}^{2}$ above baseline. $^{30}$ Hundza et al. ${ }^{31}$ proposed a method for accurately and reliably detect gait cycle in PD. They used gyroscope angular rate reversal to identify the start of each gait cycle during walking. By interpolating zerocrossing of angular rate from a foot mounted IMU, they detect the termination of forward swing (TOFS) and consider it as physically close to heel strike. They define it as the start and end point of the stride time for each gait cycle. In our case, the strategy was to determine the feasibility of using an inertial sensor as a heel switch alternative, in order to trigger and adapt the stimulation. We aimed to detect stationary periods from the foot mounted IMU combining accelerometer and gyrometer measurements. For defining lowest sensibility thresholds, we had to firstly filter raw inertial data. As the latency was a crucial parameter, we chose to use an Exponential Moving Average (EMA, low pass, Infinite Impulse Response - IIR) filter. At any time, output of the filter is a weighted sum of the new sensor value and the old filter output. Filter coefficient controls the filtering effects:

$$
\begin{gathered}
g \mathrm{X}_{\text {filt }}=(1-\alpha) * g \mathrm{X}_{\text {filt old }}+\alpha * g \mathrm{X}_{\text {raw }} \\
\text { with } \alpha \in[0,1]
\end{gathered}
$$

Our data being processed at $100 \mathrm{~Hz}$, we designed a low pass filter of order 1 type butterworth with an attenuation of $3 \mathrm{~dB}$ at a cutoff frequency of $5 \mathrm{~Hz}$ using $\alpha$ $=0.1367$ with only one sample late.

In motionless situation, the acceleration norm is supposed to be around $a c c_{t h}=9.81 . \mathrm{m}_{\mathrm{s}} \mathrm{s}^{-2}$ (depending on sensor quality and calibration). From gyrometer angular profile, we determined a magnitude threshold, which is the limit between foot flat phase and heel off phase $\left(g y r_{t h} \cong 30 \mathrm{deg} / \mathrm{s}\right)$.

Based on Equ. 2, stimulation was triggered when a non-stationary period was detected.

$$
\text { if }\left\{\begin{array}{c}
\operatorname{norm}\left(a c c_{\text {filt }}\right) \leq a c c_{t h} \\
\text { and } \\
\text { norm }\left(\operatorname{gyr}_{\text {filt }}\right) \leq g y r_{t h}
\end{array}\right\} \Rightarrow>\text { stationary state }
$$

As shown in Fig 2, post-processing of angular speed combined with stimulation start-stop events clearly demonstrates that stimulus was sent during heel-off phase, which was the initial goal.

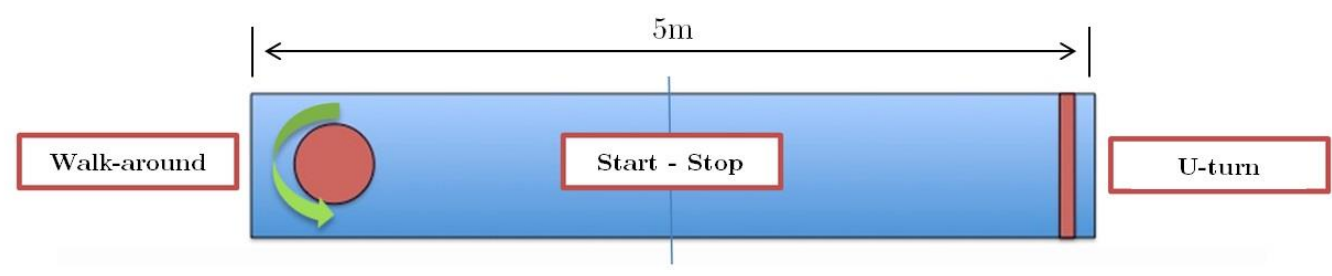

Fig 4. Five meters experimentation path. The subject starts in the middle of the walkway, walks 2,5m, performs a uturn at the line. goes back $5 \mathrm{~m}$. walks around a cone. walks back $2.5 \mathrm{~m}$ then stops where he started. 


\section{Sensitive electrical stimulation based cueing in Parkinson's disease}

Eur J Transl Myol 26 (2): 122-128

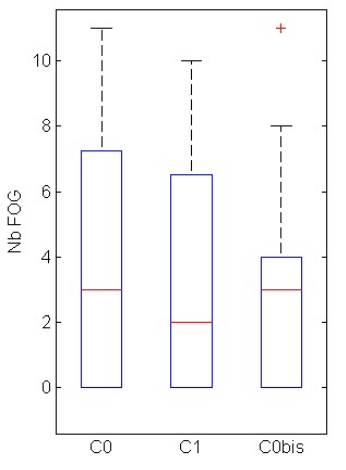

Fig 5. Number of Freezing of Gait events compared between baseline (CO), stimulation (Cl) and control baseline (CObis) on all subjects.

\section{Experimental Protocol}

13 subjects with Parkinson's disease (10 male, 3 female; Age range: 60 to 82 years) participated to the study. The protocol has been approved by the local ethical committee (international identification number NCT02317289). Participants were recruited at the Neurology (Chauliac Hospital) and Gerontology (Balmes Center) departments of Montpellier hospital (CHU Montpellier). All subjects gave their informed written consent. Subjects started from standing in the middle of a gait carpet, equipped with one HikoB@ $\odot$ Fox (HikoB@ Villeurbanne, France) inertial measurement unit strapped to the foot and a wirelessly programmable electro-stimulator (Phenix @) Neo Usb, Montpellier, France) strapped around the shank. Two electrodes were set up on the foot as shown in Fig. 1. After a short familiarization to walk under stimulation, subjects were instructed to walk towards a line draw on the ground, then do a U-turn, walk 5 meters, walk around a cone and keep walking to the start-stop line in the middle of the carpet. The test was repeated five times under the following conditions: no cueing ( $\mathrm{C} 0$ ), stimulation cueing (C1), no cueing (CObis). C0 is considered as the baseline. For eliminating learning bias, we asked the subject to perform a 10-min ecological path (random walk in the hospital) between $\mathrm{C} 1$ and Cobis conditions.

\section{Results}

In each condition, we analyzed the last three repetitions. Each FOG event, u-turn execution times, 5meters execution times and times to walk-around the cone were quantified from video recordings. In a previous study, ${ }^{32}$ we observed that subjects scored with a high occurrence of FOG in daily life were not necessarily those who were the most subject to FOG during clinical experimental protocol. In table 1, we can see subject 2 and 4 were reported as frequent freezers in their daily life meanwhile they did not freeze in $\mathrm{C} 0$. Thus we chose to classify the results between subjects who did experienced FOG in $\mathrm{C} 0$ and those who did not "freeze".

In "freezers" group $(n=9)$, we observed that cueing globally decreased of $12 \%$ FOG occurrence compared to baseline without cueing (Fig. 5). Table 2 shows cueing's effects in relation to baselines for the total group and in each subgroup during the different experimental path phases. In every tasks on both "freezers" and "non-freezers" subjects, Table 2 shows that cueing improved gait performances. Considering

Table 1 Clinical profiles of subjects who participated in the study.

\begin{tabular}{|c|c|c|c|c|c|c|c|c|}
\hline ID & AGE & $\begin{array}{c}\text { DISEASE } \\
\text { DURATION }\end{array}$ & $\begin{array}{c}\text { STAGE } \\
(\mathrm{H} \& \mathrm{Y})\end{array}$ & $\begin{array}{c}\text { AGE } \\
\text { OF } \\
\text { ONSET }\end{array}$ & $\begin{array}{c}\text { *UPDRS } \\
\text { 3.11: FREEZING } \\
\text { / 3.10: WALK / } \\
\text { GLOBAL PART } \\
\text { III }\end{array}$ & $\begin{array}{c}\text { Freezing } \\
\text { (Occasional / } \\
\text { Frequent) }\end{array}$ & $\begin{array}{l}\text { Falls } \\
(\mathrm{Y} / \mathrm{N})\end{array}$ & *MOCA \\
\hline 1 & 71 & 5 & 2 & 57 & $1 / 1 / 28$ & $\mathrm{O}$ & $\mathrm{N}$ & 26 \\
\hline 2 & 63 & 7 & 3 & 53 & $1 / 1 / 28$ & $\mathrm{~F}$ & $\mathrm{Y}$ & 30 \\
\hline 3 & 71 & 18 & 3 & 53 & $2 / 2 / 40$ & na & na & na \\
\hline 4 & 74 & 22 & 3 & 52 & $1 / 2 / 23$ & $\mathrm{~F}$ & $Y$ & 25 \\
\hline 5 & 72 & 7 & 3 & 65 & 2/2/na & $\mathrm{F}$ & $\mathrm{Y}$ & 27 \\
\hline 6 & 74 & 8 & 3 & 48 & na & na & $\mathrm{Y}$ & 12 \\
\hline 7 & 60 & 13 & 3 & 47 & 3/na/na & $\mathrm{F}$ & $\mathrm{Y}$ & 25 \\
\hline 8 & 66 & 3 & 4 & 63 & na & $\mathrm{F}$ & $\mathrm{Y}$ & 23 \\
\hline 9 & 76 & 7 & 3 & 69 & na & $\mathrm{F}$ & $\mathrm{N}$ & 23 \\
\hline 10 & 74 & 10 & 3 & 64 & $2 / 3 / 35$ & $\mathrm{~F}$ & $\mathrm{~N}$ & 21 \\
\hline 11 & 66 & 14 & 4 & 52 & na & na & na & na \\
\hline 12 & 74 & 13 & 3 & 61 & $2 / 2 / 41$ & $\mathrm{~F}$ & $\mathrm{Y}$ & 25 \\
\hline 13 & 82 & 15 & 3 & 47 & $1 / 3 / 47$ & $\mathrm{~F}$ & $\mathrm{Y}$ & 26 \\
\hline
\end{tabular}

*UPDRS: Unified Parkinson's Disease Rating Scale, from 0(normal) to 4(inability)

*MOCA: Montreal Cognitive Assesment, the total possible score is 30 points; a score of 26 or above is considered normal. 
Table 2. Durations (standard deviation) of U-turn, 5-meters and Walk-around phases compared between baseline 1 (C0), stimulation (C1) and baseline 2 (CObis) for the total group ( $(n=13)$ and subgroups (freezers and non-freezers in CO).

\begin{tabular}{|c|c|c|c|}
\hline & $\begin{array}{c}\text { All } \\
(\mathrm{N}=13)\end{array}$ & $\begin{array}{c}\text { Non Freezers } \\
(\mathrm{n}=4)\end{array}$ & $\begin{array}{c}\text { Freezers } \\
(n=9)\end{array}$ \\
\hline \multicolumn{4}{|c|}{ U-Turn Time (s) } \\
\hline Baseline $1(\mathrm{C} 0)$ & $3.0(1.6)$ & $1.9(0.6)$ & $4.1(3.0)$ \\
\hline Stimulation $(\mathbf{C} 1)$ & $2.6(1.1)$ & $1.8(0.6)$ & $3.4(2.7)$ \\
\hline Baseline 2 (CObis) & $3.2(1.3)$ & $2.2(0.9)$ & $4.1(3.0)$ \\
\hline \multicolumn{4}{|c|}{ Walk Around Time (s) } \\
\hline Baseline $1(\mathrm{C} 0)$ & $4.7(3.5)$ & $2.2(0.3)$ & $7.2(3.5)$ \\
\hline Stimulation $(\mathrm{C} 1)$ & $3.8(2.3)$ & $2.1(0.4)$ & $5.4(4.1)$ \\
\hline Baseline 2 (C0bis) & $4.7(3.5)$ & $2.2(0.3)$ & $7.2(6.5)$ \\
\hline \multicolumn{4}{|c|}{$5 \mathrm{~m}$ Time $(\mathrm{s})$} \\
\hline Baseline $1(\mathrm{C} 0)$ & $6.7(1.8)$ & $5.4(1.3)$ & $7.9(4.2)$ \\
\hline Stimulation (C1) & $5.8(1.3)$ & $4.9(1.6)$ & $6.7(2.1)$ \\
\hline Baseline 2 (CObis) & $6.6(1.6)$ & $5.4(0.8)$ & $7.7(3.3)$ \\
\hline
\end{tabular}

all participants, we observed a reduction of $15 \%$ in turning time, $14 \%$ in $5-\mathrm{m}$ covering duration and $19 \%$ in time needed to walk-around the cone. In "freezers" subgroup, turning time is improved by $21 \%$, time to walk-around the cone is reduced of $25 \%$ and the duration needed to cover the 5-m walk decreased of $18 \%$. The entire path is completed $19 \%$ shorter than baseline.

\section{Discussion}

Through this study, we investigated the feasibility of using electrical stimulation as a cueing method in Parkinson's Disease. The aim was to investigate the capability of this cueing modality to prevent or at least reduce FOG events and to improve gait performances. As partly related to environmental triggers, freezing of gait is difficult to evaluate during a clinical protocol. Based on previous observations and literature, we designed a protocol including turning phases in order to increase FOG events occurrence. This hypothesis has been validated as FOG repartition on all trials was four times more frequent during turning phases than when walking in a straight line. Results show a global positive effect on gait performances, as the time needed to achieve the protocol was considerably shorter with stimulation cueing. "Freezer" subjects tend to be more responsive to cueing, with a turning time improved by $21 \%$. We also observe a $12 \%$ decrease in FOG occurrence compared to baseline. However, our subject population was too small for showing a statistically significant effect. Subjects reported no discomforts with electrical stimulation sensation. Some of them expressed an interest in such a possibility to be helped while walking in their daily-life and seemed to accept the additional technological equipment coming with it. We noticed an important range between subjects regarding minimum electrical intensity needed for feeling the stimulus. Such as auditory and visual stimuli in other studies, the electrical cueing responsivity seemed also to be clearly disparate depending on the subjects. Among our 13 participants, stimulation had a strong significant effect on two (respectively $70 \%$ of FOG events reduction and a 5-m path $45 \%$ shorter compared to baseline), while it did not affect at all some others. In the last case, stimulation cueing never worsen performances or FOG occurrence. In this protocol, the use of an inertial sensor based trigger did not offer much more functionality than a basic heel switch. However, having access to gait kinematics data, ${ }^{29,33}$ and to path information from only one sensor could be useful to real-time adapt cueing, when for example a turning phase or a FOG event ${ }^{17,32}$ is detected by the sensor. We could also modulate stimulation or dynamically change the trigger timing. Many other triggering strategies could be investigated and some technical aspects need to be improved for getting rid of some latency problems we experienced during the trials.

In conclusion, his study suggests a new sensitive cueing modality based on electrical stimulation for Parkinson Disease population. Experimental results brought to light a favorable effect in both gait performances and FOG occurrence. Improvements of our cueing strategy have still to be investigated and discussed, but this preliminary work demonstrated encouraging results. A larger study is also required to statistically support these findings and to compare them to other cueing methods usually applied in PD.

\section{Contributions}

$\mathrm{BS}$ is the principle author and responsible for the algorithm and software design and data analysis. CA and BS carried out the experiments. DA provided his electrical stimulation devices technical expertise for implementing the experimental protocol. CG was the medical doctor. Helped by $\mathrm{CV}$, they were in charge of designing the protocol accepted by the ethical committee, subject evaluation and inclusion. All the 


\section{Sensitive electrical stimulation based cueing in Parkinson's disease}

Eur J Transl Myol 26 (2): 122-128

authors read and approved the final version of the manuscript.

\section{Acknowledgement}

This work was supported by an INRIA internal financial support: ADT SENSBIO and a Montpellier Hospital internal financial support (AOI PARKDEMAR CHU Montpellier).

\section{Conflict of Interest}

The authors declare no potential conflict of interests.

\section{Corresponding Author}

Benoît Sijobert, INRIA - LIRMM Université de Montpellier, Montpellier, France.

E-mail: benoit.sijobert@inria.fr

E-mails of coAuthors

Christine Azevedo: christine.azevedo@inria.fr.

David Andreu: david.andreu@inria.fr

Claudia Verna: c-verna@chu-montpellier.fr

Christian Geny: c-geny@chu-montpellier.fr

\section{References}

1. Vandenbossche J, Deroost N, Soetens E, et al. Freezing of gait in Parkinson's disease: disturbances in automaticity and control. Front Hum Neurosci 2012:356.

2. Jankovic J. Parkinson' s disease : clinical features and diagnosis. Neurol Neurosurg Psychiatry 2008;79:368-76.

3. Bloem BR, Hausdorff JM, Visser JE, Giladi N. Falls and freezing of Gait in Parkinson's disease: A review of two interconnected, episodic phenomena. Mov Disord 2004;19:871-84.

4. Heremans E, Nieuwboer A, Vercruysse S. Freezing of gait in Parkinson's disease: where are we now? Curr Neurol Neurosci Rep 2013;13:350.

5. Moore ST, MacDougall HG, Gracies JM, Cohen HS, Ondo WG. Long-term monitoring of gait in Parkinson's disease. Gait Posture 2007;26:200-7.

6. Moore ST, MacDougall HG, Ondo WG. Ambulatory monitoring of freezing of gait in Parkinson's disease. J Neurosci Methods 2008;167:340-48.

7. Rahman S, Griffin HJ, Quinn NP JM. The factors that induce or overcome freezing of gait in Parkinson's disease. Behav Neurol 2008;19:12736.

8. Rubinstein TC, Giladi N, Hausdorff JM. The power of cueing to circumvent dopamine deficits: A review of physical therapy treatment of gait disturbances in Parkinson's disease. Mov Disord 2002;17:1148-60.

9. Jovanov E, Wang E, Verhagen L, Fredrickson M, Fratangelo R. deFOG - A real time system for detection and unfreezing of gait of Parkinson's patients. Proc 31st Annu Int Conf IEEE Eng Med Biol Soc Eng Futur Biomed EMBC 2009 2009;5151-4.
10. Baram Y. Virtual Sensory Feedback for Gait Improvement in Neurological Patients. Front Neurol 2013;4(October):138.

11. Suteerawattananon M, Morris GS, Etnyre BR, Jankovic J, Protas EJ. Effects of visual and auditory cues on gait in individuals with Parkinson's disease. J Neurol Sci 2004;219:63-9.

12. Thaut MH, McIntosh GC, Rice RR, Miller R a, Rathbun J, Brault JM. Rhythmic auditory stimulation in gait training for Parkinson's disease patients. Mov Disord 1996;11:193-200.

13. Spaulding SJ, Barber B, Colby M, Cormack B, Mick T, Jenkins ME. Cueing and gait improvement among people with Parkinson's disease: A meta-analysis. Arch Phys Med Rehabil 2013;94(3):562-70. http://dx.doi.org/10.1016/j.ap mr.2012.10.026

14. Mann GE, Finn SM, Taylor PN. A pilot study to investigate the feasibility of electrical stimulation to assist gait in Parkinson's disease. Neuromodulation 2008;11(2):143-9.

15. Djurić-Jovičić M, Radovanović S, Petrović I, Azevedo C, Mann G, Popović MB. The impact of functional electrical stimulation (FES) on freezing of gait (FOG) in patients with Parkinson's disease. Clin Neurophysiol 2013 http://linkinghub.elsevier.com/retrieve/pii/S13882 45712008218

16. Schaafsma JD, Balash Y, Gurevich T, Bartels AL, Hausdorff JM, Giladi N. Characterization of freezing of gait subtypes and the response of each to levodopa in Parkinson's disease. Eur J Neurol 2003;10:391-8.

17. Coste CA, Sijobert B, Pissard-Gibollet R, Pasquier M, Espiau B, Geny C. Detection of freezing of gait in Parkinson disease: preliminary results. Sensors (Basel) 2014;14:6819-27. http://www.ncbi.nlm.nih.gov/pubmed/24740014

18. Plotnik M, Giladi N, Balash Y, Peretz C, Hausdorff JM. Is freezing of gait in Parkinson's disease related to asymmetric motor function? Ann Neurol 2005;57:656-63.

19. Crenna P, Carpinella I, Rabuffetti M, et al. The association between impaired turning and normal straight walking in Parkinson's disease. Gait Posture 2007;26:172-8.

20. Nieuwboer A, Baker K, Willems A-M, et al. The short-term effects of different cueing modalities on turn speed in people with Parkinson's disease. Neurorehabil Neural Repair 2009;23:831-6.

21. Nieuwboer a, Giladi N. The challenge of evaluating freezing of gait in patients with Parkinson's disease. $\mathrm{Br}$ J Neurosurg 2008;22:S16-8. http://www.ncbi.nlm.nih.gov/pubmed/19085348

22. Vaugoyeau M, Viel S, Assaiante C, Amblard B, Azulay JP. Impaired vertical postural control and proprioceptive integration deficits in Parkinson's 


\section{Sensitive electrical stimulation based cueing in Parkinson's disease}

Eur J Transl Myol 26 (2): 122-128

disease. Neuroscience 2007;146:852-63. doi.org/10.1016/j.neuroscience.2007.01.052

23. Mesure S, Azulay JP, Pouget J, Amblard B. Strategies of segmental stabilization during gait in Parkinson's disease. Exp Brain Res 1999;129:57381.

24. Khudados E, Cody FWJ, O'Boyle DJ. Proprioceptive regulation of voluntary ankle movements, demonstrated using muscle vibration, is impaired by Parkinson's disease. J Neurol Neurosurg Psychiatry 1999;67:504-10. doi/10.1136/jnnp.67.4.504

25. El-Tamawy MS, Darwish MH, Khallaf ME. Effects of augmented proprioceptive cues on the parameters of gait of individuals with Parkinson's disease. Ann Indian Acad Neurol 2012;15:267-72.

26. Kleiner A, Galli M, Gaglione $M$, et al. The Parkinsonian Gait Spatiotemporal Parameters Quantified by a Single Inertial Sensor before and after Automated Mechanical Peripheral Stimulation Treatment. Parkinsons Dis 2015;2015.

27. Spaich EG, Svaneborg N, Jørgensen HR, Andersen OK. Rehabilitation of the hemiparetic gait by nociceptive withdrawal reflex-based functional electrical therapy: a randomized, single-blinded study. J Neuroeng Rehabil 2014;11:81. http://www.ncbi.nlm.nih.gov/pubmed/24885645

28. Muro-de-la-Herran A, García-Zapirain B, MéndezZorrilla A. Gait analysis methods: An overview of wearable and non-wearable systems, highlighting clinical applications. Sensors (Switzerland) 2014;14:3362-94.

29. Sijobert B, Benoussaad M, Denys J, Pissardgibollet $\mathrm{R}$, Coste CA. Implementation and validation of a stride length estimation algorithm , using a single inertial sensor on healthy and Parkinson' $\mathrm{s}$ disease subjects. Health (Irvine Calif) $2015 ; 13: 1-7$. doi.org/10.4236/health.2015 .76084

30. MacDougall HG, Moore ST. Marching to the beat of the same drummer: the spontaneous tempo of human locomotion. J Appl Physiol 2005;99:1164-73.

31. Hundza SR, Hook WR, Harris CR, et al. Accurate and reliable gait cycle detection in parkinson's disease. IEEE Trans Neural Syst Rehabil Eng 2014;22(1):127-37. http://www.ncbi.nlm.nih.gov/ pubmed/24158491

32. Sijobert B, Denys J, Coste CA, Geny C. IMU based detection of freezing of gait and festination in Parkinson's disease. In: 2014 IEEE 19th International Functional Electrical Stimulation Society Annual Conference, IFESS 2014 Conference Proceedings. 2014.

33. Benoussaad M, Sijobert B, Mombaur K, Azevedo-Coste C. Robust Foot Clearance Estimation based on Acceleration Integration of Mounted Inertial Measurement Unit. Sensors 2015;(October 2015):1-13. 